\title{
Ultra Low Power Bandgap Strom- und Spannungsquellen in CMOS-Technologie für integrierte drahtlose Systeme
}

\author{
T. Fedtschenko, R. Kokozinski, and S. Kolnsberg
}

Fraunhofer-Institut für Mikroelektronische Schaltungen und Systeme (IMS), Finkenstraße 61, D-47057 Duisburg, Germany

Zusammenfassung. In modernen drahtlosen Systemen sind niedriger Stromverbrauch und das Betreiben bei niedriger Spannung (Low Voltage Operation) von entscheidender Bedeutung. Dabei ist für viele elektronische Anwendungen eine genaue Spannungs- bzw. Stromreferenz notwendig. Aus diesem Grund werden an eine Referenzquelle hohe Anforderungen bezüglich ihrer Temperatur- und Langzeitstabilität gestellt, was gleichzeitig schwierig mit den "Low-PowerÄnforderungen zu vereinbaren ist.

Besonders für die auf passiven Transpondern basierenden RFID-Systeme, bei denen die Energieversorgung der Schaltung aus dem Hochfrequenzträgersignal gewonnen wird, stellt die Erzeugung einer genauen Spannungsreferenz ein Problem dar. Vor allem die Spannungsstabilität und die Unabhängigkeit von Temperatur- und Prozessschwankungen bereiten große Schwierigkeiten.

In diesem Artikel wird das Design einer CMOS Bandgap Strom- und Spannungsreferenz, realisiert in einer $0,25 \mu \mathrm{m}$ CMOS-Prozess-Technologie, mit 2,5 V Versorgungsspannung vorgestellt. Die entwickelte Schaltung hat eine Stromaufnahme von $50 \mu \mathrm{A}$ bei einer Genauigkeit von $1 \%$ im Temperaturbereich von $-40^{\circ} \mathrm{C}$ bis $125^{\circ} \mathrm{C}$. Ein Testchip wurde in die Fertigung eingespeist. Ausgehend von einem Überblick über bekannte Realisierungen von Bandgap-Schaltungen wird die Besonderheit der neu entwickelten Low-Power Schaltung vergleichend gegenübergestellt.

\section{Einleitung}

Der Bedarf nach batteriebetriebenen tragbaren Elektronikgeräten erfordert die Herstellung von bei niedriger Leistungsaufnahme und Versorgungsspannungen arbeitenden Systemen, um die Lebensdauer einer Batterie zu erhöhen. Das

Correspondence to: T. Fedtschenko

(tatjana.fedtschenko@ims.fraunhofer.de)
Betreiben bei niedriger Spannung gewährt eine höhere Leistungsfähigkeit und Integrationsdichte.

Die Tendenz zur Miniaturisierung der CMOS-Strukturen hat auch eine Senkung der nominalen Versorgungsspannungen zur Folge. In einer 0,5 $\mu \mathrm{m}$ CMOS-Prozess-Technologie beträgt die nominale Versorgungsspannung $3,3 \mathrm{~V}$; für $0,25 \mu \mathrm{m}$ CMOS ist es $2,5 \mathrm{~V}$, für $0,15 \mu \mathrm{m}$ CMOS ist die Versorgungsspannung $1,5 \mathrm{~V}$ und wird mit jeder neuen Prozessgeneration sinken. Es muss die Bedingung

$V_{D D}+\left|V_{S S}\right| \geq V_{t N}+\left|V_{t P}\right|$

erfüllt werden, wobei $V_{D D}$ und $V_{S S}$ eine positive und eine negative Versorgungspannung ist, $V_{t N}$ und $V_{t P}$ Schwellenspannungen entsprechend des NMOS- und des PMOSTransistors sind (Allen et al., 1995).

Das Problem ist, dass die Schwellenspannungen der Transistoren nicht in gleichem Maß wie die Versorgungsspannungen sinken und damit die Erfüllung der Gleichung (1) erschwert wird.

Die Genauigkeit der Referenzspannung hat häufig eine erhebliche Auswirkung auf die Leistungsfähigkeit des Chips. So ist die Präzisions-Bezugsspannungsquelle ein wesentlicher Bestandteil fast aller elektronischen Systeme. Zum Beispiel wird die Auflösung eines analog-digital- oder digitalanalog Konverters durch die Präzision der Spannungsreferenz über den ganzen Versorgungsspannung- und Betriebstemperaturbereich begrenzt. Somit ist es erforderlich, dass Spannungsreferenzen höhere Versorgungsspannungsunterdrückungen und niedrigere Temperaturkoeffizienten aufzuweisen haben.

Eine weit verbreitete Lösung für die Erzeugung einer temperaturunabhängigen Spannung ist die Bandgapreferenz. Aus der Addition zweier Spannungen, einer mit negativer und einer anderen mit positiver Temperaturabhängigkeit, resultiert eine stabile temperaturunabhängige Spannung. Bekannt ist, dass die Basis-Emitter Spannung $V_{B E}$ eines bipolaren Transistors zur absoluten Temperatur (CTAT) fast kom-

Published by Copernicus GmbH on behalf of the URSI Landesausschuss in der Bundesrepublik Deutschland e.V. 


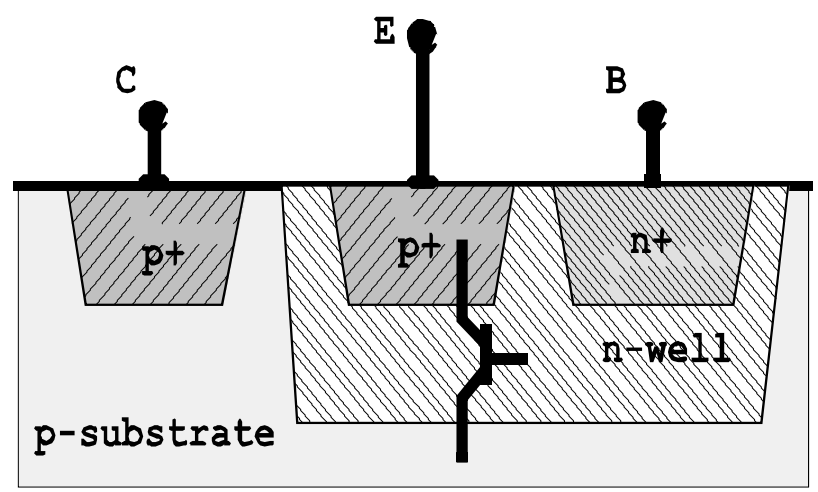

Abbildung 1. Realisierung des PNP Transistors in CMOS - Technologie

plementär ist, d.h. sie verringert sich linear mit der steigenden Temperatur (Razavi, 2001). Die Basis-Emitter Spannung $V_{B E}$ extrapoliert zu absolutem Null gleicht in der Näherung erster Ordnung der Bandgapspannung des Halbleiters, $V_{G 0}$. Wird eine Spannung mit dem Betrag $V_{B E}$ aber direkt proportional zur absoluten Temperatur (PTAT) mit der Spannung $V_{B E}$ addiert, bekommen wir eine definierte Spannung, die von der Temperatur unabhängig ist.

Um auf diese Weise eine Spannungsreferenz zu erstellen, werden bipolare Transistoren gebraucht. In Anbetracht der Kostenfrage werden solche Lösungen erforderlich, die Schaffung einer Referenz ohne Gebrauch von einem kostspieligen BiCMOS-Prozess ermöglichen.

Allerdings ist die Implementierung solcher Spannungsquellen bei niedrigen Versorgungsspannungen und hohen Schwellenspannungen sehr schwierig.

In den reinen CMOS-Prozessen sind frei anschließbare bipolare Transistoren nicht vorhanden. Dennoch kann eine bipolare Bandgapreferenz in eine CMOS Bandgap-Referenz umgesetzt werden. Dafür werden die parasitären Wannentransistoren verwendet (Razavi, 2001), die vertikale bipolare Transistore mit der Wanne als Basis und mit dem Substrat als Kollektor bilden, wie in der Abb. 1 illustriert wird. Bei solchen Substrat-PNP-Transistoren in der CMOS-Technologie wird vorausgesetzt, dass der Kollektor am Substratpotential liegt. Die PNP-Transistoren werden als geerdete Diode angeschlossen, in der ein Emitter Anode ist und die Kathode - die Basis - mit dem Kollektor (Substrat) zusammen gebunden ist.

\section{Realisierung der Bandgap-Referenz Schaltung}

Das versorgungsspannungsunabhängige Biasing Schema kann mit bipolaren Transistor-Core kombiniert werden, wie in der Abb. 2 dargestellt wird. Angenommen, dass M1-M2 und M3-M4 identische Paare sind, ist $V_{B E}$ der Spannungsabfall über einem bipolaren Transistor der wie eine in Vorwärts-

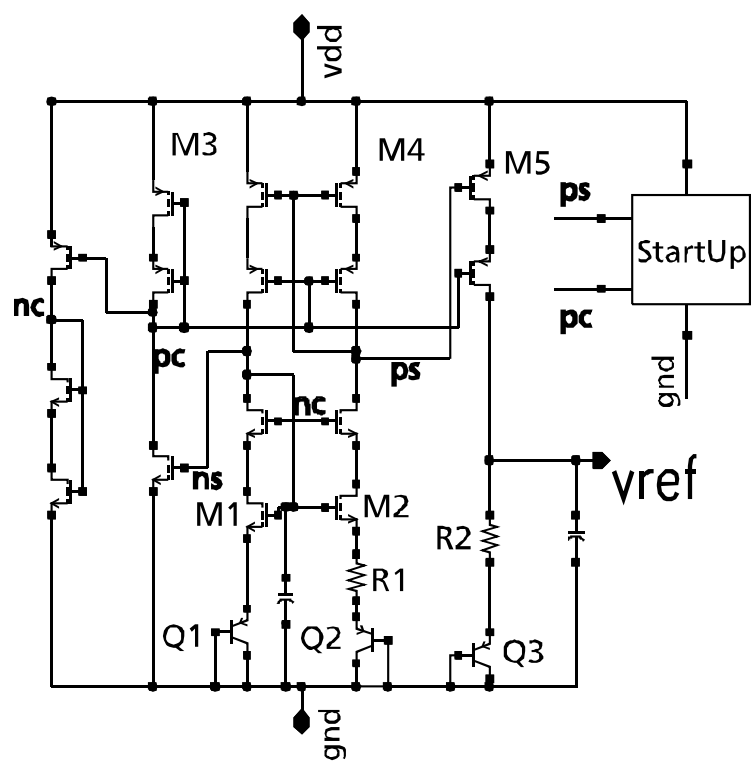

Abbildung 2. Bandgap Spannungsquelle.

richtung betriebene Diode verbunden wird:

$V_{B E}=V_{T}$

$\triangle V_{B E}=V_{B E 1}-V_{B E 2}=V_{T} \ln N$,

$I_{D 1}=I_{D 2}=\frac{\Delta V_{B E}}{R_{1}}=\frac{V_{T} \ln N}{R_{1}}$,

wo $N$ das Stromdichteverhältnis der beiden Bipolartransistor Q2 zu Q1 ist. Der Strom $I_{D 5}$ verhält sich identisch. Wird die PTAT-Spannung $I_{D 5} R_{2}$ einer Basis-Emitter-Spannung hinzugefügt (vorausgesetzt, dass alle PMOS -Transistoren identisch sind), folgt daraus:

$V_{R E F}=V_{B E 3}+\frac{R_{2}}{R_{1}} V_{T} \ln N$.

Es ist anzumerken, dass die vorangegangene Analyse nur bei Vernachlässigung der Kanal-Längenmodulation korrekt ist. In der Praxis kann sie bedeutende Versorgungsspannungsabhängigkeit verursachen. Um diesen Effekt zu minimieren, wird in jedem Stromzweig sowohl eine NMOS- als auch eine PMOS-Low-Voltage-Cascodetopologie verwendet. Abbildung 3 zeigt die simulierten Charaktereigenschaften der Bandgap-Referenz. Bei der Versorgungsspannung von $3 \mathrm{~V}$ bis zu $5 \mathrm{~V}$ beträgt die Versorgungsspannungsabhängigkeit der implementierten Spannungsreferenz $\pm 5 \mathrm{mV}$. Die Temperaturvariation wurde für eine Versorgungsspannung von $3,3 \mathrm{~V}$ und eine Temperaturspanne $-40^{\circ} \mathrm{C}$ bis $125^{\circ} \mathrm{C}$ simuliert. Die Schaltung erreicht einen Temperaturkoeffizienten von $10 \mathrm{ppm} /{ }^{\circ} \mathrm{C}$. Diese Schaltung wurde für einen drahtlosen Drucktransponder in einer 1,2 $\mu \mathrm{m}$ CMOS-ProzessTechnologie realisiert und in die Praxis umgesetzt. Der gemessene Stromverbrauch dieser Schaltung liegt unterhalb von $7 \mu \mathrm{A}$ für eine Versorgungsspannung 3,3 V. 


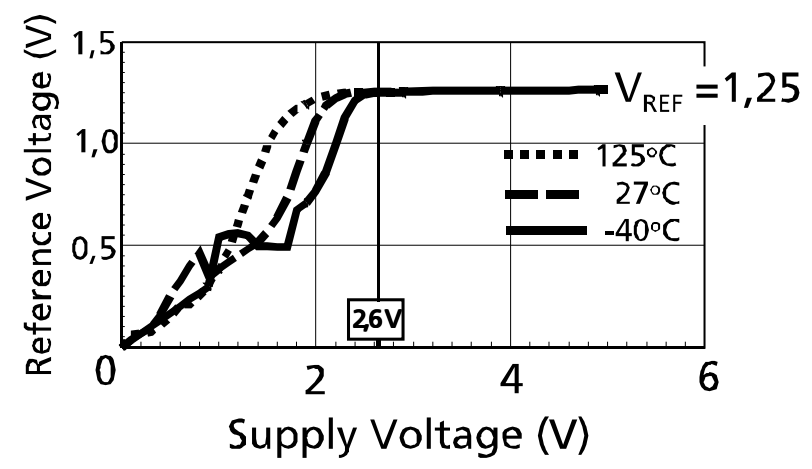

Abbildung 3. Verhalten der Bandgap-Spannungsreferenz für den Drucktransponder

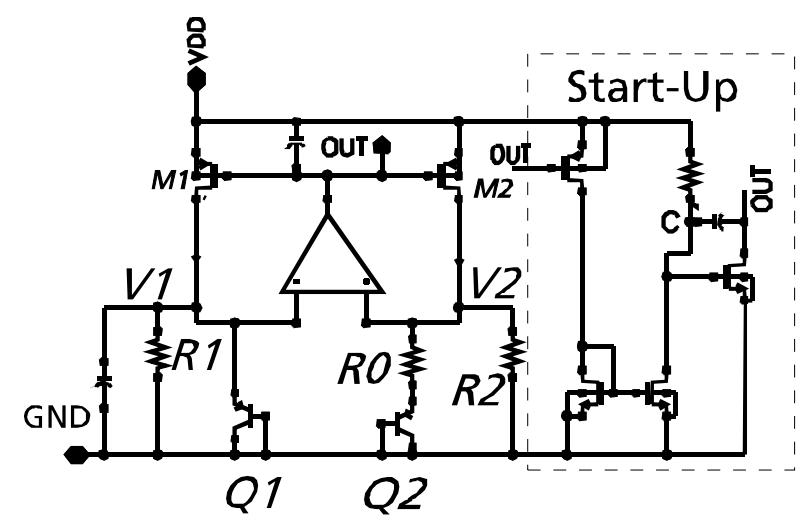

Abbildung 4. Subbandgap-Referenz

Der Grafik ist zu entnehmen, dass die Versorgungsspannung nicht unterhalb 2,6 V fallen darf, was im Widerspruch zur Low-Voltage Tendenz steht. Eine der wenigen Lösungen dieses Problems ist die in Banba et al. (1999) berichtete Subbandgap-Referenz.

\section{Realisierung der Subbandgap-Referenz Schaltung}

Die Lösung basiert auf dem in der Abb. 4 gezeigtem Widerstand-Teiler. Da die Spannungen $V 1$ und $V 2$ durch den Operationsverstärker geregelt werden, sind die Ströme in den nominal gleichen Widerständen $R 1$ und $R 2$ proportional zu $V_{B E}$. Folglich werden die Gates von M1, M2 und M3 wie Stromspiegel an einen allgemeinen Knoten angeschlossen, damit die Ströme $I 1, I 2$ und $I 3$ den gleichen Wert annehmen. Die Ausgangsspannung der vorgeschlagenen BandgapReferenz $V_{R E F}$ wird

$V_{R E F}=R 4\left(\frac{V_{B E}}{R 2}+\frac{d V_{B E}}{R 3}\right)$.

Die Weiten der Transistoren M1-M2 wurden ziemlich groß gewählt, um das $1 / f$ Rauschen herabzusetzen. Die Temperaturabhängigkeit der benutzten Widerstände wurde durch

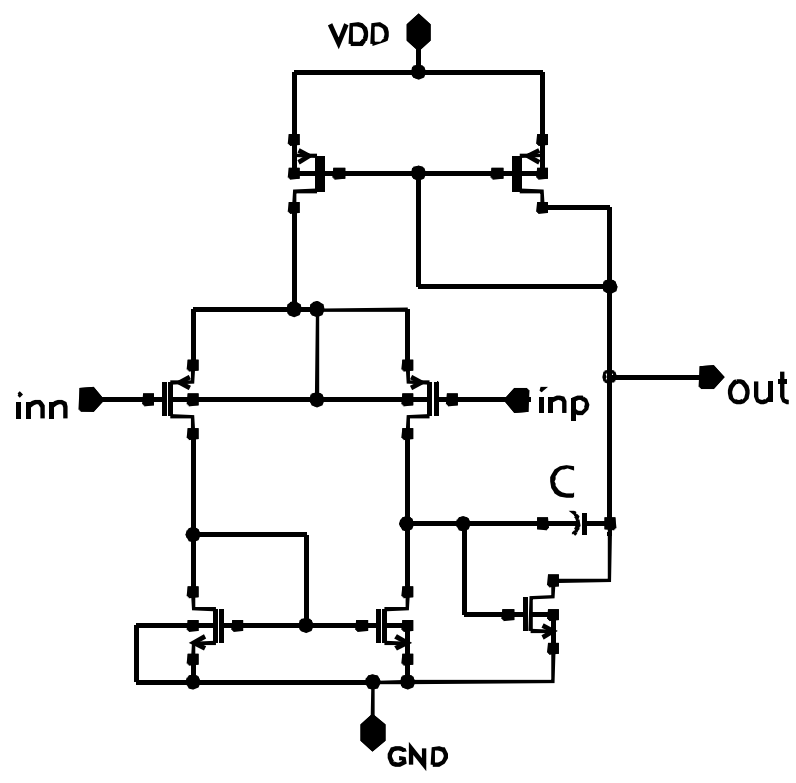

Abbildung 5. Operationsverstärker

Einsetzen des gleichen Materials ausgeglichen. Widerstände wurden als einfache Poly-Widerstände realisiert, da in diesem Prozess kein hoch-ohmscher Poly-Widerstand vorhanden ist und die NWanneWiderstände nicht ausreichende Genauigkeit liefern. Große Widerstandswerte sind erforderlich, um den trotz der niedrigen Leistungsaufnahme hohen Spannungsabfall über den Widerständen zu gewährleisten.

Die Schaltung in der Abb. 4 kann prinzipiell mit einer sehr niedrigen Versorgungsspannung funktionieren, da nur eine in Vorwärtsrichtung betriebene Diode und ein PMOSTransistor untergebracht werden müssen. Jedoch entstehen einige Probleme in der Implementierung des Operationsverstärkers (OPAMP), der im richtigen Arbeitspunkt betrieben werden muss (Pierazzi, Andrea, et al., 2001.) Es muss sichergestellt werden, dass diese minimale Spannung für den zuverlässigen Betrieb des Operationsverstärkers ausreichend ist. Low-Voltage Realisierungen der Referenzschaltungen werden hauptsächlich durch die Spannungsanforderungen des Operationsverstärkers eingeschränkt. So sollte das Design des Operationsverstärkers die notwendige Verstärkung bei niedrigen Versorgungsspannungen liefern können und damit die Hauptentwurfsrichtlinie sein.

Die Eingangsstufe des OPAMPs (Abb. 5) wird mit PMOS-Transistoren realisiert, um die Einganggleichtaktspannung und die niedrige $V_{B E}$-Spannung aufeinander abzustimmen. Die Bulkanschlüsse beider Eingangs-PMOSTransistoren werden mit ihren Sourceanschlüssen verbunden, um den Body-Effekt und infolgedessen die übermäßig hohen Schwellenspannungen zu eliminieren. Wenn die Versorgungsspannung unterhalb $1,6 \mathrm{~V}$ fällt, treten die Eingangstransistoren in die schwache Inversion ein. Trotzdem funktioniert die Bandgap-Referenz richtig, solange die Ver- 


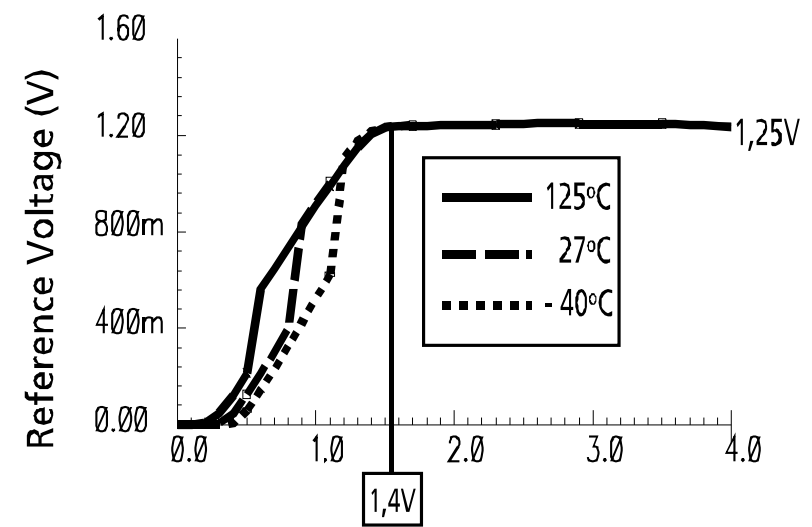

Supply Voltage (V)

Abbildung 6. Ausgangsspannung der Subbandgap-Referenz

sorgungsspannung mindestens $1,4 \mathrm{~V}$ beträgt. Unterhalb dieses Wertes ist die OPAMP-Verstärkung nicht mehr ausreichend, um einen richtigen Referenzwert zu garantieren. Um den Offset-Effekt zwischen den zwei Eingangstransistoren des OPAMPs zu mindern, sollte die Verstärkung so groß wie möglich sein, deshalb wurde ein zweistufiger Verstärker verwendet. Die Verstärkung des implementierten OPAMPs ist über $60 \mathrm{~dB}$. Um die Stabilität des OPAMPs zu sichern und ausreichende Phasenreserve zu gewährleisten, wurde ein Miller-Kondensator $C$ eingesetzt. Um Missmatch und Offset-Effekte zu verringern, wurden Langkanaltransistoren benutzt.

Die Variation der Bezugsspannung der SubbandgapReferenz wird in der Abb. 6 grafisch dargestellt. Die Temperaturvariation wurde für einen Temperaturbereich von $40^{\circ} \mathrm{C}$ bis zu $125^{\circ} \mathrm{C}$ mit einer Versorgungsspannung 2,5 V simuliert. Die Schaltung erzielt einen Temperaturkoeffizienten von $15 \mathrm{ppm} /{ }^{\circ} \mathrm{C}$. Die Versorgungsspannungsabhängigkeit ergibt $\pm 2,5 \mu \mathrm{V}$ für einen Bereich von $1,4 \mathrm{~V}$ bis zu $6 \mathrm{~V}$.

In manchen Fällen ist es sinnvoll einen Referenzstrom anstatt einer Referenzspannung zur Verfügung zu stellen. Anders als Spannungen weisen Ströme keinen Abfall entlang einer langen Metalleitung auf. Folglich werden Stromreferenzen in komplexen analogen Schaltungen verwendet, wo häufig lange Metallleitungen vorkommen. Das für die Stromreferenz eingesetzte Designprinzip ist mit dem für die Spannungsreferenz äquivalent. Um eine temperaturunabhängige Stromreferenz hervorzubringen, sollte das Verhältnis $m$ von $R 1 \mathrm{zu} R 0$ folgendem Zusammenhang entsprechen (Jiwei Chen et al., 2003)

$m=\frac{q B_{B E 1, T 0}}{k T_{0} \ln N}\left(\frac{\beta+\gamma}{\alpha-\gamma}\right)$

wo $\alpha=1 / T_{0}, \beta$ - der Diodentemperaturkoeffizient ist; und $\gamma$ - der Widerstandstemperaturkoeffizient ist.

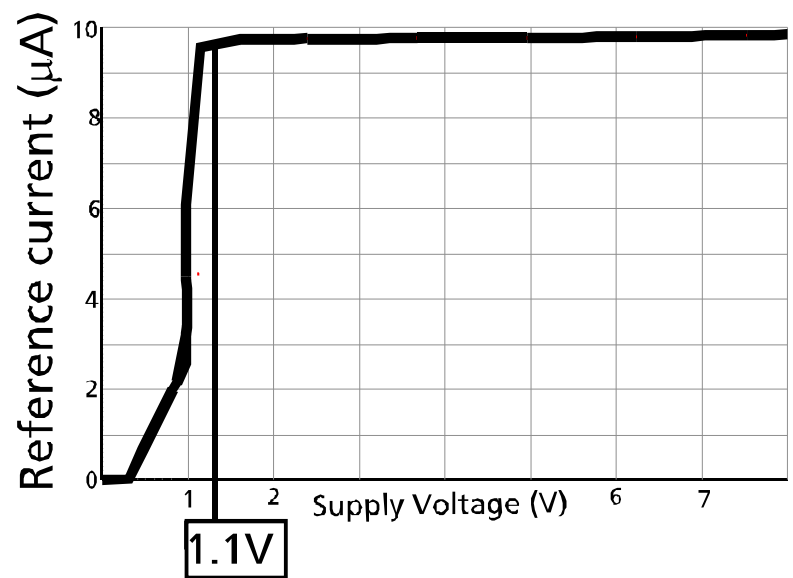

Abbildung 7. Vesorgungsspannungsabhängigkeit der Stromreferenz

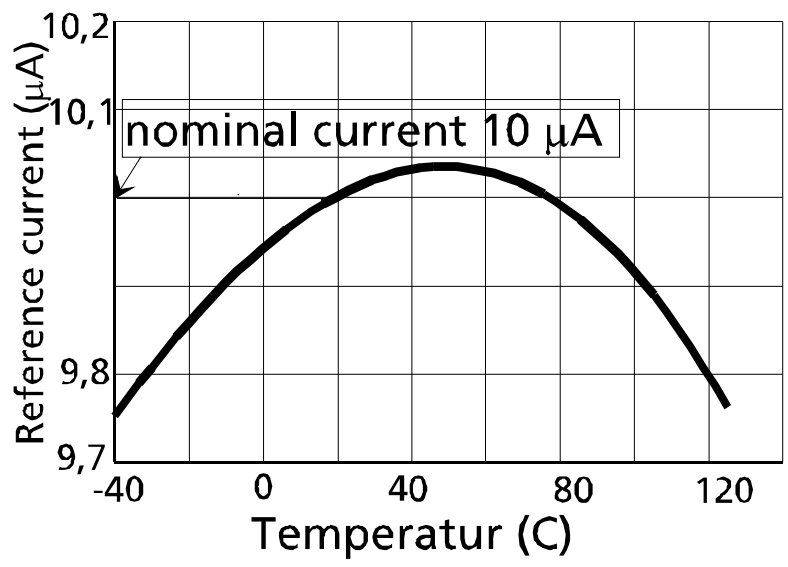

Abbildung 8. Temperaturabhängigkeit der Stromreferenz

Abbildung 7 zeigt die simulierte Abhängigkeit der Stromreferenz von der Versorgungsspannung bei $27^{\circ} \mathrm{C}$. Man kann erkennen, dass diese Schaltung sich im richtigen Arbeitspunkt befindet, solange die Versorgungsspannung höher als $1,1 \mathrm{~V}$ ist. Der Strom bleibt konstant bei Spannungen höher als $1,1 \mathrm{~V}$, dabei wird ein niedriger Spannungskoeffizient von 0,6\% erzielt. Die Temperatureigenschaft des Ausgangsstromes dieser Schaltung wird in der Abb. 8 dargestellt. Es ist festzustellen, dass der Temperaturkoeffizient des $10 \mu \mathrm{A} \mathrm{Re}-$ ferenzstromes den Wert $170 \mathrm{ppm} /{ }^{\circ} \mathrm{C}$ im Temperaturbereich von $-45^{\circ} \mathrm{C}$ zu $125^{\circ} \mathrm{C}$ erreicht. Die Stromaufnahme für beide Referenzschaltungen liegt unterhalb $50 \mu \mathrm{A}$ bei der Versorgungsspannung von 2,5 V.

Diese Schaltungen wurden in ein Design für Low-Power Wireless Transceiver, realisiert in einer $0,25 \mu \mathrm{m}$ CMOSProzess-Technologie, implementiert. Die Abb. 9 präsentiert die Layouts der implementierten Referenzen. Die Fläche der Spannungsreferenz beträgt ca. $270 \mu \mathrm{m} \times 100 \mu \mathrm{m}$, die der Stromreferenz beträgt ca. $210 \mu \mathrm{m} \times 100 \mu \mathrm{m}$. 
Tabelle 1.

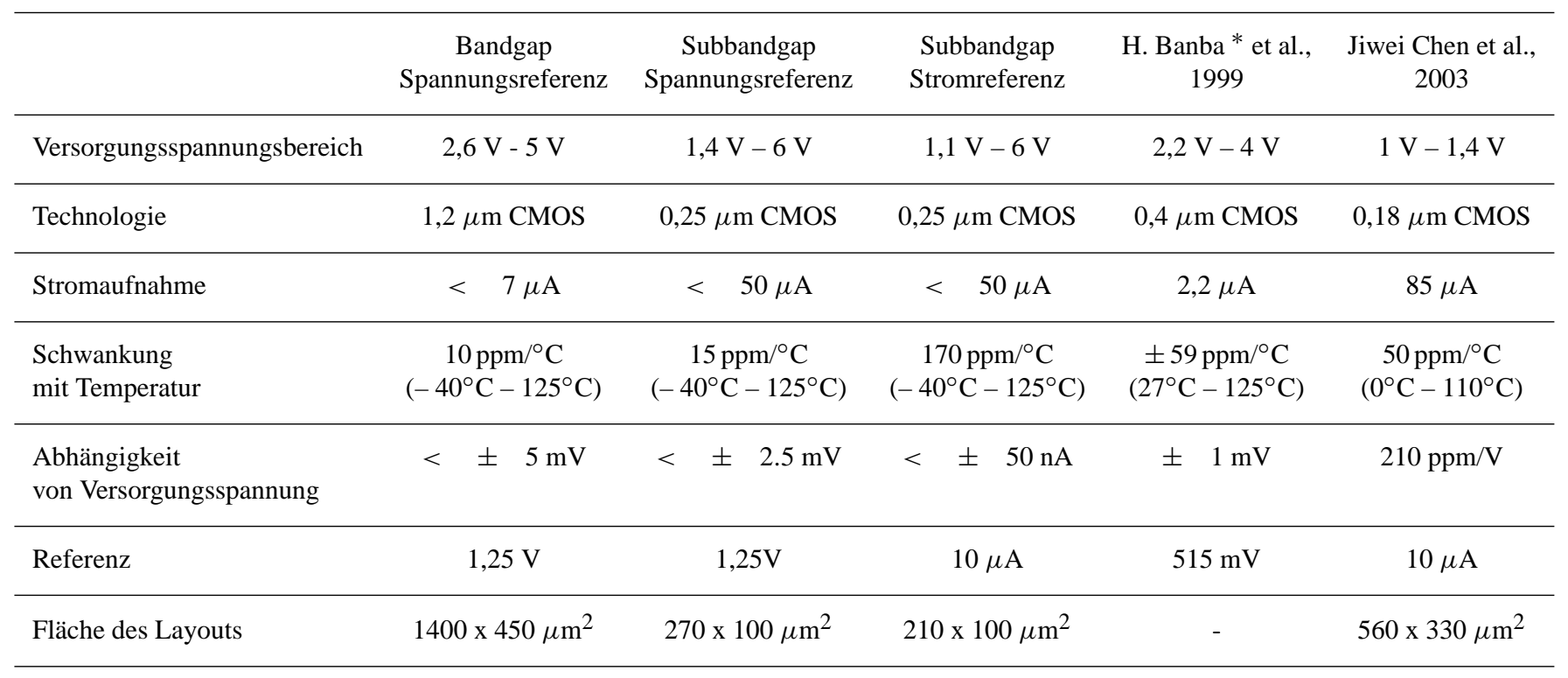

* Die native NMOS-Transistoren wurden verwendet

\section{Zusammenfassung und Ausblick}

Dieser Artikel befasst sich mit Low-Voltage Low Power Designtechniken für Strom- und Spannungreferenzen. Die Bandgapquellen wurden dargestellt, die bei der Versorgungsspannung ab 1,4 V stabile temperaturunabhängige Referenzen liefern und dabei weniger als $50 \mu \mathrm{A}$ Strom verbrauchen. Außerdem wurde ein Bandgapdesign vorgestellt, das erlaubt, die Stromaufnahme unterhalb $7 \mu \mathrm{A}$ zu halten. In der Tabelle 1 werden die simulierten Ergebnisse zweier Testchips zusammengefasst.

In diesem Artikel wurde das Rauschen in den Stromund Spannungsreferenzen nicht berücksichtigt. Obwohl eine Bandgap-Referenz selbst nicht eine signalverarbeitende Schaltung ist, könnte das Rauschen an ihrem Ausgang für die Signalverarbeitung sehr nachteilig sein. Störanfälligkeit ist in vielen Designs kritisch, folglich ist eine Rauschcharakteristik für Low-Voltage Bandgap-Referenzen in zunehmendem Maße wichtig und ist bei weiteren Redesing-Maßnahmen zu berücksichtigen.

\section{Literatur}

Razavi, B.: Design of Analog CMOS Integrated Circuits, New York, 2001.

Allen, P. E., Blalock, B. J., and Rincon, G.A.: A 1V CMOS Opamp Using Bulk-Driven MOSFET's, Proc. 1995IEEE ISSCC, 192-

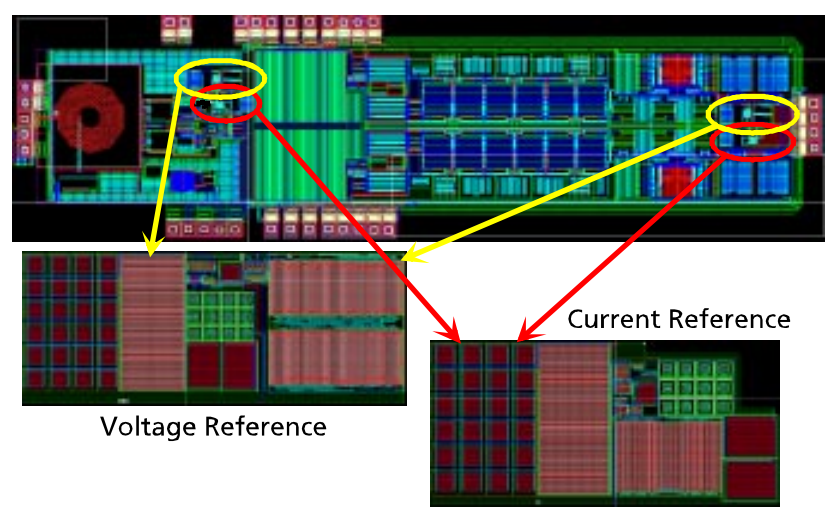

Abbildung 9. Testchip. Die Größe der Spannungsreferenz ist $270 \times 100 \mu \mathrm{m}^{2}$, die der Stromreferenz ist $210 \times 100 \mu \mathrm{m}^{2}$

193, 1995

Banba, H., Shiga, Hi., Umezawa, A., Miyaba, T., Tanzawa, T., et al.: A CMOS Bandgap Reference Circuit with Sub 1V Operation, IEEE J. of Solid State, Circuits, 34, 670-674, 1999.

Pierazzi, A., Boni, A., and Morandi, C.: Bandgap Reference for near $1 \mathrm{~V}$ operation in standard CMOS Technology, IEEE2001- Custom Integrated Circuits Conf., 463-466, 2001.

Jiwei Chen and Bingxue Shi: New approach to CMOS current reference with very low temperature coefficient, GLSVLSI'03, Washington, DC, USA, 2003. 